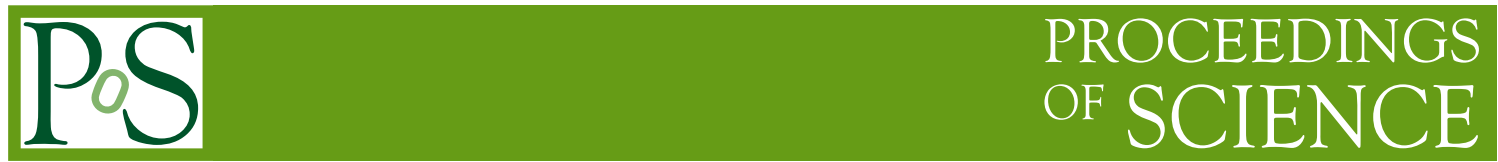

\title{
Extra Dimensions in Photon or Jet plus Missing Transverse Energy
}

\author{
Marco CARDACI* on behalf of the CMS Collaboration \\ Universiteit Antwerpen \\ E-mail: marco.cardaci@cern.ch
}

Recent studies of the CMS collaboration are presented on the sensitivity to searches for large (ADD) extra dimensions in channels with missing transverse energy (MET), i.e. the channels jets plus MET and photon plus MET. These studies are based on detailed detector simulation, including all Standard Model backgrounds. Particular emphasis is given to possible early discoveries, i.e. with $100 \mathrm{pb}^{-1}$ or less. Projected $95 \% \mathrm{CL}$ exclusion limits as function of luminosity are presented.

European Physical Society Europhysics Conference on High Energy Physics

July 16-22, 2009

Krakow, Poland

* Speaker. 


\section{Introduction}

The Standard Model (SM) of Elementary Particles has been tested at a high level of precision by many accelerator- and non accelerator-based experiments, which have made it one of the major cornerstone of physics. Nevertheless, the SM is an effective theory valid up to a cut-off energy, affected by a pathology known as hierarchy problem, a puzzling difference between the Electroweak $\left(\sim 10^{2} \mathrm{GeV}\right)$ and the Planck $\left(\sim 10^{19} \mathrm{GeV}\right)$ scales. The Arkani-Hamed, Dimopoulos, Dvali (ADD) model [1] of extra dimensions solves the hierarchy problem introducing $\delta$ extra spatial dimensions, which in the simplest scenario are compactified over a torus and all have the same radius $R$. In this model the Planck scale becomes an effective scale related to the more fundamental scale $M_{D}$ via the relation: $M_{D}^{\delta+2} R^{\delta} \sim M_{P l}^{2}$. Consequences of the ADD model are that the extra dimensions can be 'macroscopic' for TeV-ish $M_{D}$ scales and be tested at colliders such as Tevatron and LHC. Gravitons interact 'weakly' with the ordinary SM particles and escape detection: therefore, two clear ADD signatures of the direct graviton production, which will be probed with CMS detector [2], are a large missing transverse energy (MET) together with high transverse momentum jet or photon.

\section{Search for $\gamma+$ MET at $14 \mathrm{TeV}$}

The topology of this signature is an energetic photon recoiling back to back with a large MET vector. SM processes which can mimic this signature are: $\mathrm{Z}+\gamma$ with $\mathrm{Z}$ decaying into neutrinos (irreducible background), the leptonic $\mathrm{W}+$ jets decay, $\mathrm{W}(e v)$ and $\mathrm{W}(\tau(e v v) v)$, QCD multijets, $\gamma+$ jets, $\mathrm{Z}+$ jets with $\mathrm{Z}$ into neutrinos and $\gamma \gamma$ production. Both the signal and the SM backgrounds were processed with the CMS fast simulation. To reject the background a set of cuts is foreseen: MET $>400 \mathrm{GeV}$ and $p_{T}$ of most energetic jet (jet1) larger than $400 \mathrm{GeV}$, with the jet contained in the range $|\eta|<2.4$, a veto against tracks with $p_{T}>40 \mathrm{GeV}, \Delta \phi(\gamma$, MET $)>2.5$ and identification of the photon thanks to an isolated photon likelihood $>0.2$. The irreducible background can be estimated from $\mathrm{Z}(\mu \mu / \mathrm{ee})+$ jets defining a control region with: photon within $|\eta|<2.4$ and $p_{T}$ $>400 \mathrm{GeV}$, muons with $|\eta|<2.3$ and $p_{T}>20 \mathrm{GeV}$, electrons with $|\eta|<2.4, p_{T}>20 \mathrm{GeV}$ and electron likelihood $>0.65$ and $\mathrm{Z}$ in the mass window $80 \mathrm{GeV}$ to $100 \mathrm{GeV}, \Delta \phi(Z, \gamma)>2.5$ and $\left(p_{T}^{\gamma}-\right.$ $\left.p_{T}^{Z}\right) /\left(p_{T}^{\gamma}+p_{T}^{Z}\right)<0.25$. The control samples can be then rescaled by branching ratios, acceptance and reconstruction efficiencies. Taking into account photon energy resolution, MET reconstruction uncertainty and PDF systematics, the preliminary result is that $5 \sigma$ discovery is possible for $M_{D} \sim$ 1.0 TeV after $\mathrm{O}\left(100 \mathrm{pb}^{-1}\right)$ and $M_{D} \sim 2.0 \mathrm{TeV}$ after $\mathrm{O}\left(1 \mathrm{fb}^{-1}\right)$ for $\delta=2$.

\section{Search for Jet + MET final state at $14 \mathrm{TeV}$ and $10 \mathrm{TeV}$}

The topology of this signature is of one energetic jet back to back with a large MET vector. SM processes which can mimic this signature are: $\mathrm{Z}+$ jets with $\mathrm{Z}$ decaying into neutrinos (irreducible background), $\mathrm{W}+\mathrm{jets}$ followed by a leptonic $\mathrm{W}$ decay, QCD multijets and $t \bar{t}+\mathrm{jets}$ and single top production. For this analysis the signal was generated with SHERPA generator [3] version 1.0.11, at $14 \mathrm{TeV}$, in 18 different samples, with $M_{D}$ ranging from 2 to $7 \mathrm{TeV}$ and $\delta=2,3,4$ and simulated with the CMS fast simulation, while the W/Z/t $t \bar{t}+$ jets were generated with ALPGEN 2.12 [3], QCD multijets with PYTHIA 6.409 [3] and simulated with the CMS full simulation. The optimal set 

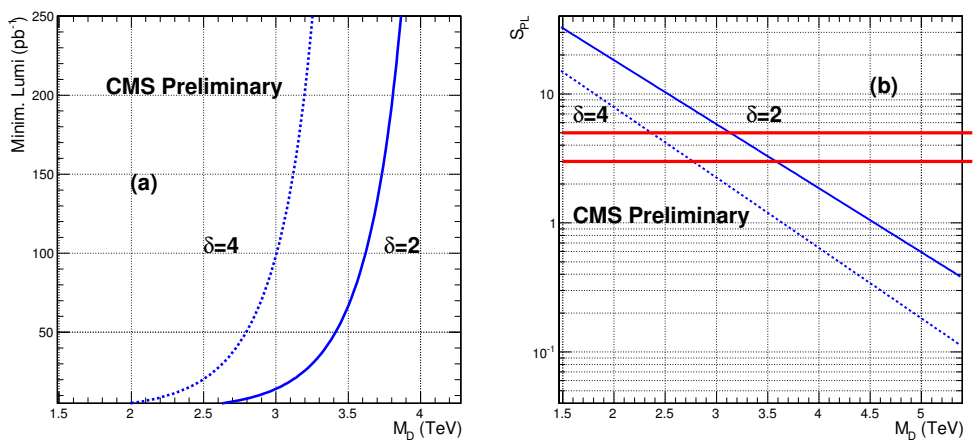

Figure 1: (a): $95 \% \mathrm{CL}$ exclusion limits in terms of integrated luminosity as a function of $M_{D}$ and $\delta$. (b): Discovery potential as a function of $M_{D}$ for $200 \mathrm{pb}^{-1}$. All estimates are for Monojet at $10 \mathrm{TeV}$ LHC energy.

of selection cuts for this analysis was found to be: MET $>400 \mathrm{GeV}$, most energetic jet $p_{T}>350$ $\mathrm{GeV}$ for jet with $|\eta|<1.7$, Jet ElectroMagnetic Fraction (JEMF) $<0.9$, no isolated tracks with $p_{T}>15 \mathrm{GeV}$ in a cone of radius equal to 0.3 , finally the azimuthal cuts $\Delta \phi$ (MET,jet1) $>2.8$ and $\Delta \phi($ MET,jet2) $>0.5$ which are very effective to reduce the QCD multijets events. A data-driven technique has been prepared and tested which will allow to estimate the irreducible background from data, $\mathrm{Z}(v v)+$ jets. This technique will rely on an almost pure $\mathrm{W}(\mu v)+$ jets sample selected by requiring a well isolated lepton. The sample will be scaled by theoretical (W/Z ratio) and instrumental (trigger and isolation efficiency) factors after signal selections are applied. Taking into account all systematic uncertainties such as the PDF dependence using CTEQ6M, the potential of discovery was evaluated using the significance estimator $S_{p L}$ [4]. A $5 \sigma$ discovery reach can be achieved with $M_{D}=3.1$ (2.2) $\mathrm{TeV}$ for $\delta=2$ (4) after $\mathrm{O}\left(100 \mathrm{pb}^{-1}\right.$ ) (see also [5]). This analysis was repeated at $10 \mathrm{TeV}$ center-of-mass energy. The new analysis exploits corrected jets and MHT, the vectorial sum of the jets above a certain threshold, which replaces the MET in all the analysis. This variable is expected to be less affected by instrumental effects than MET. The set of cuts chosen for this analysis is: MHT $>250 \mathrm{GeV}$, for jets with $p_{T}>50 \mathrm{GeV}$ and $|\eta(j e t)|<3,0.1<\mathrm{JEMF}<$ $0.9, p_{T}$ (jet1) $>200 \mathrm{GeV}, \mid \eta($ jet 1$) \mid<1.7$, and all the other cuts as considered in the analysis at $14 \mathrm{TeV}$. Considering all the systematic uncertainties as in the previous analysis the conclusion (see also Fig. 1) is that after $200 \mathrm{pb}^{-1}, M_{D}$ can be excluded better than 3.8(3.2)TeV for $\delta=2(4)$. Also early discoveries for $\delta=2(4)$ are possible, if $M_{D}$ is below 3.1(2.3) TeV, respectively (see also [6]).

\section{References}

[1] N. Arkani-Hamed et al., Physics Letters B429, 263 (1998)

[2] CMS Collaboration, JINST 0803 (2008) S08004

[3] SHERPA: T. Gleisberg et al. JHEP 0402, 056 (2004); ALPGEN: M. L. Mangano et al. JHEP 0307, 001 (2003); PHYTIA: T. Sjöstrand et al. Comput. Phys. Commun. 135238 (2001)

[4] R. D. Cousins et al., Nucl. Instr. A595, 480

[5] CMS Collaboration, PAS EXO-09-013

[6] CMS Collaboration, PAS EXO-08-011 